\section{DOUBLE BLIND PLACEBO CONTROLLED FOOD CHALLENGE USEFUL TO DISCONFIRM OVER ESTIMATED DIAGNOSIS OF CMPA IN CHILDREN}

S. Accomando, F. Serraino, R. Antona, A. Ferlisi, V. Pellitteri, S. Leone, F. Matina, A. Liotta, G. Corsello

University of Palermo, Palermo, Italy

Background and aims: The incidence of CMPA in infancy seems to be approximately $3 \%$. In Italy diagnosis of CMPA is often over estimated. The double-blind, placebo controlled food challenge(DBPCFC) is widely considered as the "gold standard" for the diagnosis of food allergy.

Methods: 14 patients, (12 months-12 yrs) previously diagnosed as having CMPA, underwent our diagnostic algorithm in order to confirm or to exclude diagnosis. Diagnostic algorithm includes: total blood cell count, serum IgE assay, RAST, betalactotest, Prick by prick with fresh milk, chemical examination and eosinophilic cell count of the stools. DBPCFC was performed with extensively hydrolyzed formula (as placebo) VS a lactose-free, cow milk derived formula.

At discharge a post challenge form was given to parents to record delayed symptoms that might occur at home.

Results: 12 out of 14 patients had positive results for skin prick test (prick by prick) and four out of them also presented specific IgE against CMPs. 13 patients showed tolerance to CMP during and after DBPCFC and had no symptoms also in the next $72 \mathrm{~h}$. Only one patient, aged 30 months, had to keep CMP avoidance.

Conclusion: In our survey DBPCFC demonstrated tolerance to CMP for all but one patients. DBPCFC is effective also to disconfirm diagnosis of CMPA. Skin prick tests and serological specific lgE do not always correlate with oral tolerance test.

Subjects with positive skin prick test and high serological specific lgE against CMP but tolerant at DBPCFC may reintroduce cow milk proteins in to their dietary meals.

\section{ETIOLOGY OF BRONCHIOLITIS IN A HOSPITALIZED PEDIATRIC POPULATION: PROSPECTIVE MULTICENTER STUDY}

\author{
H. Rodrigues ${ }^{1}$, N. Silva ${ }^{2}$, C. Ferreira ${ }^{3}$, \\ F. Carvalho ${ }^{4}$, H. Ramalho ${ }^{5}$, F. Branca ${ }^{6}$, \\ H. Antunes ${ }^{2,7}$
}

${ }^{1}$ Pediatrics, Unidade Local de Saúde do Alto Minho, EPE, Viana do Castelo, ${ }^{2} P e d i a t r i c s$, Braga Hospital, Braga, ${ }^{3}$ Pediatrics, Centro Hospitalar do Médio Ave, EPE, Guimarães, ${ }^{4}$ Pediatrics, Centro Hospitalar do Alto Ave, EPE, Famalicão,

${ }^{5}$ Pediatrics, Santa Maria Maior Hospital, EPE, Barcelos, ${ }^{6}$ Clinical Pathology, Braga Hospital, 'Life and Health Sciences Research Institute (ICVS), School of Health Sciences, University of Minho, Braga, Portugal

Background and aims: In 2006, bronchiolitis due to adenovirus nosocomial infections resulted in the closure of a pediatric department in northern Portugal.

Objectives: To determine the etiology of bronchiolitis in northern Portugal.

Methods: It was a prospective multicenter study on the etiology of bronchiolitis during the respiratory syncytial virus (RSV) season (November-April). Children $\leq 24$ months of age admitted for a first wheezing episode were included. Nasopharyngeal specimens were analyzed by an indirect immunofluorescentantibody assay (IFA) for RSV, adenovirus (HAdV), parainfluenza (PIV) 1-3 and influenza (IV)Aand B and by polymerase chain reaction (PCR) or reverse transcription-PCR for the same viruses and for human metapneumovirus (hMPV), bocavirus (HBoV), rhinovirus (HRV), coronaviruses (229/E; NL63; OC43; HKU1) and enterovirus.

Results: During this period, 253 children were included, 249 IFA analyses and 207 PCRs were performed. IFA detected RSV in $58.1 \%$; PCR increased it to $66.7 \%$. IFA detected HAdV in $3.2 \%$, PCR 10.0\%. PCR detected IV A in 5; IV B in 2; PIV 1 in 6, PIV 2 in 4 and PIV 3 in 11 cases. HBoV, as single agent in 2 cases, and HRV were positive in 8 samples and hMPV in 11. With this virus panel, $19.7 \%$ remained without etiology.

Conclusions: The most frequent agent was RSV, followed by HAdV. PCR can be cost-effective and more accurate than IFA, which is crucial for HAdV 